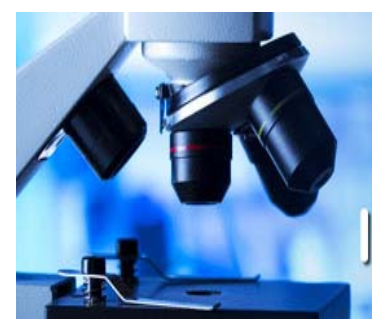

E-ISSN: 2707-4455 P-ISSN: 2707-4447 www.forensicpaper.com/ IJFM 2019; 1(2): 07-09 Received: 07-05-2019 Accepted: 10-06-2019

Dr. Shakib Hasan Department of Forensic medicine, Shaheed Suhrawardy Medical College \& Hospital, Dhaka, Bangladesh
Corresponding Author: Dr. Shakib Hasan Department of Forensic medicine, Shaheed Suhrawardy Medical College \& Hospital, Dhaka, Bangladesh

\section{To record poisoning related changes in liver autopsy}

\author{
Dr. Shakib Hasan \\ DOI: https://doi.org/10.33545/27074447.2019.v1.i2a.10
}

\begin{abstract}
Background: Acute poisoning by Pesticide compounds is a major global clinical problem, with thousands of deaths occurring every year. The present study recorded poisoning related changes in liver autopsy.

Materials \& Methods: The present study was conducted in 82 deaths due to agricultural poisons of both genders. During Autopsy detailed internal and external examinations were done and random portion of Liver were collected for histological examination and fixed in $10 \%$ formalin and stained with Hematoxylin and eosin and the findings recorded. Histological features were grouped based on degree of damage to hepatic tissue.

Results: Out of 82 patients, males were 50 and females were 32. Common poisons used was organophosphorus in 45 cases, aluminum and zinc phosphate in 13, pyrethrin compounds in 4 and unknown in 20 cases. The difference was significant $(P<0.05)$. Group I was seen in 48 , group II in 26 and III in 8 cases. The difference was significant $(P<0.05)$.

Conclusion: Authors found that maximum cases were of organophosphorus poisoning.
\end{abstract}

Keywords: organophosphorus poisoning, liver, pesticide

\section{Introduction}

It has been estimated that pesticides were directly or indirectly is responsible for more than 1 million illnesses worldwide annually ${ }^{[1]}$. Acute poisoning by Pesticide compounds is a major global clinical problem, with thousands of deaths occurring every year. Most of these pesticide poisoning and subsequent deaths occur following an intentional self-ingestion of the poison. The potential adverse impact on human health from pesticides exposure is likely to be higher due to easy availability of highly hazardous products ${ }^{[2]}$.

Three forms of phosphorus are available - white, red, and black. "Yellow phosphorus" (YP) is formed by a small amount of red phosphorus resulting discoloration of white phosphorus. YP is a general protoplasmic toxin and is used in the manufacture of fireworks, rodenticide, and fertilizers ${ }^{[3]}$ Rodenticides are available as powders or pastes containing $2 \%-5 \%$ of YP. The estimated dose of YP that is lethal to the liver is $1 \mathrm{mg} / \mathrm{kg}$, and the ingestion of that amount results in death due to acute liver failure and cardiovascular collapse ${ }^{[4]}$. Although YP poisoning may result from industrial accidents in developed countries, it also occurs in adults who are attempting suicide and accidentally by oral intake in children, particularly on festival days, in developing countries. The only definitive treatment for acute liver failure due to the ingestion of YP is liver transplantation because no antidote or medical treatment is available to reverse the toxic effects on the liver ${ }^{[5]}$. The present study recorded poisoning related changes in liver autopsy.

\section{Materials \& Methods}

The present study was conducted in the department of Forensic Medicine. It comprised of 82 deaths due to agricultural poisons of both genders. Ethical clearance was taken prior to the study.

General information such as name, age, gender etc. was recorded. During Autopsy detailed internal and external examinations were done and random portion of Liver were collected for histological examination and fixed in 10\% formalin and stained with Hematoxylin and eosin and the findings recorded. Histological features were grouped based on degree of damage to hepatic tissue. Results thus obtained were subjected to statistical analysis. P value less than 0.05 was considered significant. 


\section{Results}

Table I: Distribution of patients

\begin{tabular}{|c|c|c|}
\hline \multicolumn{3}{|c|}{ Total- 82 } \\
\hline Gender & Males & Females \\
\hline Number & 50 & 32 \\
\hline
\end{tabular}

Table I, graph I shows that out of 82 patients, males were 50 and females were 32 .

Table II: Cases detected by chemical analysis

\begin{tabular}{|c|c|c|}
\hline Poisons & Number & \multirow{2}{*}{ P value } \\
\hline OGP & 45 & \multirow{2}{*}{0.01} \\
\hline Al \& ZnPo4 & 13 & \\
\cline { 1 - 2 } Pyrethrin compounds & 4 & \\
\hline Unknown & 20 & \\
\hline
\end{tabular}

Table II shows that common poisons used was organophosphorus in 45 cases, aluminum and zinc phosphate in 13, pyrethrin compounds in 4 and unknown in 20 cases. The difference was significant $(P<0.05)$.

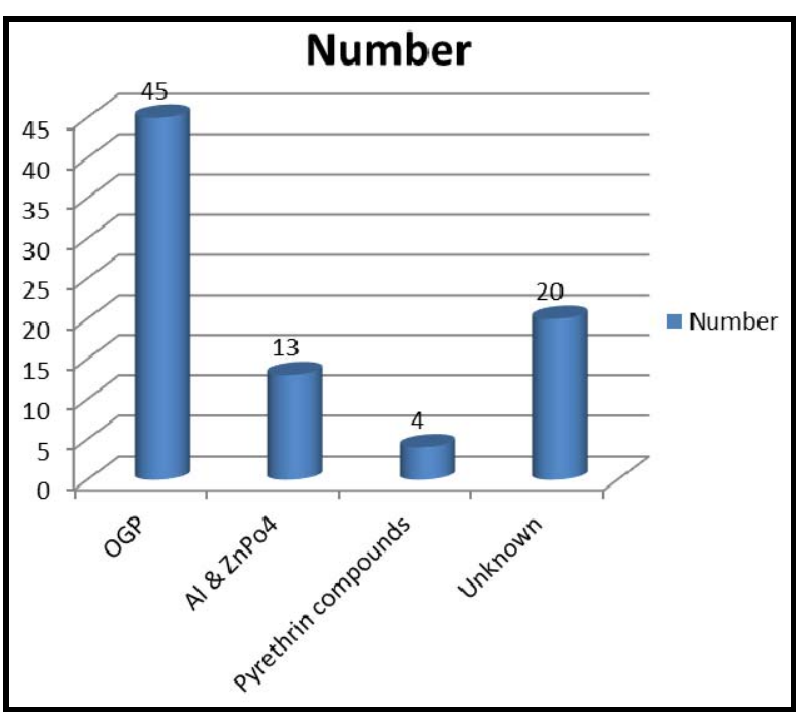

Graph I: Cases detected by chemical analysis

Table III: Histological classification of liver autopsy

\begin{tabular}{|c|c|c|}
\hline Groups & Number & P value \\
\hline Group I & 48 & \multirow{2}{*}{0.01} \\
\hline Group II & 26 & \\
\hline Group III & 8 & \\
\hline
\end{tabular}

Table III shows that group I was seen in 48 , group II in 26 and III in 8 cases. The difference was significant $(P<0.05)$.

\section{Discussion}

The incidence of poisoning cases in India is highest in the world, and it is estimated that more than 50,000 people die every year from toxic exposure ${ }^{[6]}$. The common agents causing poisoning in India appear to be pesticides (organophosphorus, organochlorine, carbamates, pyrethroids). White phosphorus is an inorganic substance used in the production of fireworks, firecrackers, ammunition, rodenticide, and fertilizers ${ }^{[7]}$. It causes direct hepatotoxicity typically resulting in periportal injury. There are several pastes and powders available in India that contain phosphorous. The usual fatal dose is approximately $1 \mathrm{mg} / \mathrm{kg}$ of body weight. Fulminant poisoning results from ingestion of more than 1 to $2 \mathrm{~g}$. ${ }^{[8]}$ The present study recorded poisoning related changes in liver autopsy.
In present study, out of 82 patients, males were 50 and females were 32 . Karki et al. ${ }^{[9]}$ conducted a study in which histological changes in liver were classified based on the degree of damage to hepatic tissue caused by agricultural poisonous substance were grouped into I, II \& III. Group I include Congestion, sinusoidal dilatation, mononuclear and neutrophillic infiltration, group II include Cytoplasmic vacuolization and hydropic degeneration, group III include Patchy or centrilobular necrosis and patchy hemorrhages. Based on duration of treatment amongst poisoning cases study group was classified. In that 653 cases were treated for less than $24 \mathrm{hr}, 174$ cases were treated for $2-5$ days, 79 cases were treated for 5- 10 days. In the present study organophosphorus compound was most commonly used and female victims in middle age group were predominant and the histopathological findings were grouped based on the degree of damage to the hepatic tissue and there incidence were identified based on type of agricultural poison and duration of treatment. Group I histological changes were predominantly seen among organophosphorus compound and the persons who were treated less than one day, group II \& III changes were predominant among phosphates, Pyrethrin compounds and the persons who were treated more than two to five days.

We found that common poisons used was organophosphorus in 45 cases, aluminum and zinc phosphate in 13, pyrethrin compounds in 4 and unknown in 20 cases. Group I changes comprise of Congestion, sinusoidal dilatation, mononuclear and neutrophillic infiltration. Group II changes has nuclear fragmentation, cytoplasmic vacuolization and hydropic degeneration and group III has patchy or centrilobular necrosis and patchy hemorrhages. We found that group I changes was seen in 48, group II in 26 and III in 8 cases.

Subhasish et al. ${ }^{[10]}$ on histopathological changes on fatal phosphine poisoning, the most frequent histopathological findings have been sinusoidal congestion and fine cytoplasmic vacuolization of hepatocytes. The fine cytoplasmic vacuoles which were the most consistent finding in our cases are rather uniform in size and shape. The vacuoles were distributed uniformly in all acinar zones in the majority $(71.1 \%)$ of cases. Other less frequent histopathological findings include portal oedema, portal vein congestion, central vein congestion, centrilobular necrosis, nuclear fragmentation, clusters of polymorphonuclear leukocytes in sinusoids macro vesicular steatosis, and sub capsular hemorrhage.

\section{Conclusion}

Authors found that maximum cases were of organophosphorus poisoning.

\section{References}

1. Subash VK, MD. Fareedullah. Current review on organophosphorus poisoning. Archives of Applied Science Research. 2010; 2(4):199-215.

2. Mauskar A, Mehta K, Nagotkar L, Shanbag P. Acute hepatic failure due to yellow phosphorus ingestion. Indian J Pharmacol. 2011; 43:355-6.

3. Pillay VV. The utility of poison control centres in diagnosing and managing cases of poisoning and overdose. J Indian Acad Forensic Med. 2010; 108(3):162-66. 
4. Senanayake N, Petris H. Mortality due to Poisoning in a Developing and Agricultural Country- Trends over 20 years. Hum Exp Toxicology. 1995; 14:808-811.

5. Vale JA. Toxicokinetic and toxicodynamic aspects of organophosphorus (OP) insecticide poisoning. Toxicol lett. 1998; 102-103:649-652.

6. Barr DB, Allen R, Olsson AO, Bravo R, Caltabiano LM, Montesano A et al. Concentrations of selective metabolites of organophosphorus pesticides in the United States population. Environ Res. 2005; 99:31426.

7. Sepideh S, Farid A. Liver histopathology of fatal phosphine poisoning Forensic Science International. 2007; 166:190-193.

8. Manish KN, Bhawna B. Pesticide poisoning - an epidemiological and histopathological study. Pacific Journal of Medical Sciences. 2013,12.

9. Karki RK, Risal A. Study of Poisoning Cases in a Tertiary Care Hospital. Kathmandu Univ Med J 2012; 10(4):70-73.

10. Subhasish S, Dipkana D. Changes in liver in case of insecticidal and alcohol poisoning: an autopsy study. Journal of Evolution of Medical and Dental Sciences. 2015; 4(27):4622-4628 